\title{
Characterization of miRNA processing machinery in the embryonic chick lung
}

\section{Rute Silva Moura ${ }^{1,2,3,}$, Patrícia Vaz-Cunha ${ }^{1,2}$, Carla Silva-Gonçalves ${ }^{1,2}$, Jorge Correia-Pinto ${ }^{1,2,4}$}

${ }^{1}$ Life and Health Sciences Research Institute (ICVS), School of Health Sciences, University of Minho, Braga, Portugal; ${ }^{2} I C V S / 3 B$ 's - PT Government Associate Laboratory, Braga/Guimarães, Portugal; ${ }^{3}$ Biology Department, School of Sciences, University of Minho, Braga, Portugal; ${ }^{4}$ Department of Pediatric Surgery, Hospital de Braga, Braga, Portugal

* Corresponding author

Running title: microRNA machinery in the developing chick lung

Key words: drosha, dgcr8, exportin-5, dicer1, chick lung

\section{ABSTRACT (250 words)}

Lung development is a very complex process that relies on the interaction of several signaling pathways that are controlled by precise regulatory mechanisms. Recently, microRNAs (miRNAs), small non-coding regulatory RNAs, have emerged as new players involved in gene expression regulation controlling several biological processes such as cellular differentiation, apoptosis and organogenesis, in both developmental and disease processes. Failure to express correctly some specific miRNAs or a component of its biosynthetic machinery during embryonic development is disastrous, resulting in severe abnormalities. Several miRNAs have already been identified as modulators of lung development. Regarding the spatial distribution of miRNAs processing machinery, only two of its members (dicer1 and argonaute) have been characterized. The present work characterizes the expression pattern of drosha, dgcr8, exportin-5 and dicer 1 in early stages of the embryonic chick lung by whole mount in situ hybridization and cross-section analysis. Overall, these genes are co-expressed in dorsal and distal mesenchyme and also in growing epithelial regions. The expression 
pattern of miRNA processing machinery supports the previously recognized regulatory role of this mechanism in epithelial and mesenchymal morphogenesis.

\section{INTRODUCTION (500-600 words)}

Pulmonary development is tightly controlled by genetically conserved intercellular signaling pathways (such as FGF, Wnt, Shh and Notch) that regulate cell proliferation, fate and differentiation, leading to branching morphogenesis and eventually to proper lung formation (reviewed by Herriges and Morrisey, 2014). More recently, microRNAs (miRNAs) arose as new players in respiratory development (Lü et al., 2005; Harris et al., 2006). In fact, several studies have reported the temporal-spatial expression of numerous miRNAs throughout lung development (reviewed by Khoshgoo et al., 2013). Moreover, miRNAs misexpression has also been described to be involved in some pulmonary diseases namely bronchopulmonary dysplasia (Bhaskaran et al., 2012) and pulmonary fibrosis (Dakhlallah et al., 2013).

MicroRNAs (miRNAs) are small single-stranded non-coding RNA fragments of about 22 nucleotides (Bartel et al., 2004) that silence gene expression by targeting specific mRNAs and therefore control innumerable cellular processes in plants and animals (Ouellet et al., 2006; Stefani \& Slack, 2008). Canonical miRNAs biogenesis is a multistep process that involves different enzymes and proteins (Nana-Sinkam et al., 2009). In most eukaryotic organisms, miRNAs are encoded in the genome as introns in protein coding genes, as independent transcription units or in polycistronic clusters (Stefani \& Slack, 2008). After being transcribed by an RNA polymerase, primary miRNAs (pri-miRNAs) are processed, still in the nucleus, by Drosha (RNase III)/DGCR8 complex originating miRNAs precursors (pre-miRNAs) with about 60-70 nucleotides. Pre-miRNAs are subsequently exported out of the nucleus by Exportin-5 (Xpo5). In the cytoplasm, these pre-miRNAs are then cleaved by Dicer (a cytoplasmatic RNase III) to form double-stranded miRNAs. The next step is the separation and selection of a mature chain of about 22 nucleotides that will associate 
with a multi-protein complex called RNA-induced silencing complex (RISC), which contain a member of the Argonaute protein family (Ouellet et al., 2006). Gene silencing occurs when this complex loaded with miRNA binds to target mRNA transcripts specifically repressing its translation, or leads to the cleavage and degradation of transcripts, compromising protein synthesis in both scenarios (Johanson et al., 2013). In addition to the canonical miRNA biogenesis pathway, there are several others miRNA biosynthesis pathways that are Drosha/DGCR8-independent or Dicerindependent (Abdelfattah et al., 2014).

miRNAs play essential roles as regulators of numerous biological processes, such as for instance cellular differentiation and apoptosis, developmental timing, immune responses, nerve system patterning and organogenesis (reviewed by Sayed and Abdellatif, 2011). Its importance is further reinforced if one considers the severe developmental abnormalities observed in dicer1 knockout mouse that leads to lethality at early embryonic developmental stages due to stem cells depletion (Bernstein et al., 2003). Regarding pulmonary development, conditional inactivation of dicer in lung epithelium leads to branching impairment and abnormal growth of the epithelial tube suggesting a crucial role for miRNA regulation in epithelial lung morphogenesis (Harris et al., 2006). Moreover, miRNA profile expression studies have demonstrated that different miRNA families are temporally expressed in embryonic and adult lung and that they modulate fetal lung development (Williams et al., 2007; Lu et al., 2007; Bhaskaran et al., 2009; Dong et al., 2010; Yang et al., 2012; Mujahid et al., 2013). Nevertheless, regarding miRNA processing machinery only dicer1 and argonaute expression patterns have been described in fetal mammalian lung (Lü et al., 2005; Harris et al., 2006).

Avian and mammalian adult lungs present a dramatically different architecture. Despite these dissimilarities the molecular events and signaling pathways underlying lung development at early stages seem to be conserved in both phyla (Moura et al., 2011; 2014). Therefore, in this study, we used the embryonic chick lung to characterize, by in 
situ hybridization, the expression pattern of drosha, dgcr8, xpo5 and dicer 1 that are essential for canonical miRNA biogenesis, in early stages of chick lung development. Furthermore, with this report we aim to fill a blank regarding the expression of miRNA processing machinery during early lung development and ultimately contribute to better comprehend the mechanisms of miRNA-dependent gene regulation.

\section{MATERIAL AND METHODS}

The work presented in this manuscript was performed in the chick model, at early stages of development, which doesn't need ethical approval from review board institution or ethics committee.

\section{Tissue collection}

Fertilized chick (Gallus gallus) eggs, obtained from commercial sources, were incubated for $4-6$ days in a $49 \%$ humidified atmosphere at $37^{\circ} \mathrm{C}$. Embryonic lungs were carefully dissected under a dissection microscope (Olympus SZX16, Japan), classified in stage b1, b2, b3 taking into account the number of secondary buds formed (Moura et al. 2011) [which corresponds to the developmental window between stage 24 to 26 , according to the Hamburger and Hamilton classification (Hamburger and Hamilton, 1992)], and then processed for whole mount in situ hybridization.

\section{In situ hybridization}

Whole mount in situ hybridization ( $\mathrm{n}=15$ per stage and probe) was performed as previously described (Henrique et al., 1995). Each set of lungs/probe was processed simultaneously and developed for the same amount of time, and then photographed with an Olympus U-LH100HG camera coupled to Olympus SZX16 stereomicroscope. Antisense digoxigenin-labeled RNA probes for drosha, dgcr8, exportin-5 and dicer1 were produced as previously described (Carraco et al., 2014).

\section{Cross-Section Preparation}

Hybridized chick lungs were fixated in paraformaldehyde $4 \%$, embedded in 2 - 
hydroxyethyl methacrylate (Heraeus Kulzer, Germany) and processed for sectioning (coronal and axial) at $25 \mu \mathrm{m}$ using a rotary microtome (Leica RM 2155, Germany). Lung sections were photographed with an Olympus DP70 camera coupled to an Olympus BX61 microscope. Afterwards, coverslips were removed, sections were rehydrated, Hematoxylin-Eosin (HE) staining was performed and the sections were photographed again.

\section{RESULTS}

\section{drosha expression pattern}

drosha mRNA is located in the mesenchyme surrounding the secondary bronchi, from the proximal most onward (Fig. 1b, dark arrowhead) and also in the distal mesenchyme (Fig. 1c, open arrowhead). On its turn, it seems to be absent in the upper epithelial and mesenchymal compartment. No differences were observed in drosha expression pattern in all stages studied. Sections of hybridized lungs confirmed the dorsal (Fig. 1e, dark arrowhead) and distal mesenchymal expression (Fig. 1f, open arrowhead). Moreover, a weak epithelial expression was observed in the main bronchus (Fig. 1f, asterisk) and in the secondary bronchi (Fig. 1d, black arrow). The expression in both cases seems to be more restricted to the inner lining of the epithelial tube. Epithelial and mesenchymal structures are clearly visible in HE stained sections (Fig. 1g-i).dgcr8 expression pattern

dgcr8 expression is evident in the dorsal mesenchyme, specifically in the region adjacent to the secondary buds (Fig. 2c, dark arrowhead), along with the main bronchus and also in the distal-medial region (Fig. 2b, open arrowhead). Mesenchymal expression seems to inaugurate with the appearance of the most proximal bud. Conversely, dgrc 8 seems to be absent from the epithelium. This pattern is maintained in the three stages studied, however a slight increase in the expression is observed from b1 to b2 stage. Sectioning of the hybridized lungs confirmed the mesenchymal expression (Fig. 2d, dark arrowhead; 2f, open arrowhead). Furthermore, it revealed a 
faint distal epithelial expression (Fig. 2f, asterisk) and also in the secondary bud epithelium (Fig. 2 d, e, black arrow). HE staining undoubtedly displays the epithelial and mesenchymal compartment (Fig. $2 g-i)$.

\section{exportin-5 expression pattern}

exportin-5 expression in the chick embryonic lung appears to be present mainly in the dorsal and distal mesenchyme (Fig. 3a, dark arrowhead; 3c, open arrowhead). Conversely, it seems absent from the proximal most mesenchymal region, and it seems that its dorsal expression initiates at the level of the first bud formed. This pattern is consistent in the three stages studied. Slide sectioning revealed that $x p o 5$ expression is not restricted to the dorsal and distal mesenchyme (Fig. $3 d$ and $3 e$, dark and open arrowhead, respectively) but it is also present in the epithelial compartment particularly in the secondary bronchi (Fig. 3d, f, black arrow) and also distally in the main bronchus (Fig. 3e, asterisk). Interestingly, in the main bronchus, the epithelial expression is absent from the proximal region, and as it occurs with mesenchymal expression, its expression begins at the level of the most proximal bud. Mesenchyme and epithelium are evident in the HE stained sections (Fig. 3g-i).

\section{dicer1 expression pattern}

dicer1 is detected primarily in the distal mesenchyme (Fig. 4a, open arrowhead) and it seems to be expressed in the epithelium at the growing tips of secondary and primary bronchi (Fig. 4). The expression pattern is, in general, similar in the three stages studied. Histological sectioning of hybridized lungs confirmed this expression pattern. It is clear that dicer1 is expressed only in the epithelium of primary and secondary bronchi (Fig. 4d, asterisk; 4f, black arrow); moreover it is possible to observe that it is also expressed in the mesenchyme surrounding the secondary buds in addition to the distal mesenchyme (Fig. 4d-f, dark arrowhead). Epithelial and mesenchymal structures are clearly visible in HE stained sections (Fig. $4 g-i)$. 


\section{DISCUSSION}

The relevance of miRNAs in fetal lung development is well recognized. miRNas have been described as key regulators of homeostasis and lung development (Lu et al., 2007), inflammation (Banerjee et al., 2010) and many pulmonary diseases namely cancer (Yanaihara et al., 2006) and chronic obstructive pulmonary disease (Sato et al., 2010); nonetheless the temporal-spatial distribution of its processing machinery remains mostly unknown. In this study we have examined transcript location, by in situ hybridization, of genes coding for the canonical biosynthesis processing machinery in chick developing lung. At early stages of chick lung development branching is monopodial, meaning that secondary buds emerge laterally off each primary bronchus (Hogan, 1999), as it occurs in early mammalian lung development (Metzger et al., 2008; Warburton et al., 2010). For this reason, only the initial stages of chick lung branching (b1 to b3) were characterized.

In general, all the components of miRNA processing machinery present a similar expression pattern. Considering the fact that these proteins function in tandem to process miRNAs their co-expression seems coherent. Their expression matches the previously described ago1/ago2 expression patterns that also contribute for the overall miRNA biosynthetic process (Lü et al., 2005).

drosha and $d g c r 8$ are mainly expressed in the dorsal and distal mesenchyme at the level of the first secondary bud formed and they are absent from the most proximal region of the lung. This might indicate a possible regulatory role in branching process by guiding mesenchymal morphogenesis. In both cases, only a faint epithelial expression, restricted to the inner lining of the epithelial tube, is observed.

xpo5 and dicer 1 are clearly expressed in the mesenchymal compartment (dorsally, from the proximal most bud onward, and distally) but also in the epithelium (mainly in the apical side). In the murine lung, dicer1 is expressed in the distal mesenchyme and epithelium (Lü et al., 2005) which is in agreement with the results obtained for the chick 
lung. It is known that dicer plays an important role in lung epithelial morphogenesis since dicer conditional knockout mice lungs fail to branch properly (Harris et al., 2006). The same authors provide evidence of a regulatory mechanism based on FGF10 signaling. It seems that the mesenchymal expression of FGF10, a chemotactic factor for lung epithelial outgrowth, is somehow regulated by miRNAs produced by the epithelium (Harris et al., 2006). Additional studies have suggested that miR-17-92 cluster, expressed in the mesoderm and endoderm (apical region), promotes the proliferative and undifferentiated state of lung epithelial progenitor cells, at early stages of lung development (Lu et al., 2007). Further studies have shown that, in the pseudoglandular stage, mir-17 family of miRNA modulates FGF10-FGFR2b downstream signaling hence regulating E-Cadherin expression, which in turn modulates epithelial bud morphogenesis in response to FGF10 signaling (Carraro et al., 2009). Taking into account that the expression pattern of both dicer1 and fgf10 (Moura et al., 2011) in the developing chick lung is similar to their mammalian counterparts, one can speculate that this regulatory mechanism is probably preserved in the avian model. Additionally, the mesenchymal expression of the microRNA processing machinery correlates with the expression of several miRNAs specifically expressed in this compartment such as miR-326, which is known to be regulated by Shh activity (Jiang et al., 2014), and miR-142-3p that contributes to the suitable proliferation of mesenchymal progenitors by controlling the level of WNT signaling (Carraro et al., 2014).The vast majority of miRNAs are synthesized by the canonical processing machinery that requires the microprocessor components (Drosha and DGCR8) to generate precursor miRNA, Exportin-5 to export it to the cytoplasm, and Dicer to form mature miRNA (Ouellet et al., 2006). In the embryonic chick lung these four genes are expressed in the mesenchyme, mainly from the most proximal bud onwards, which might indicate that canonical miRNA biogenesis pathway is probably involved in mesenchymal morphogenesis by regulating the expression of specific genes. 
On the other hand, epithelial expression is somewhat different and the four genes do not colocalize: xpo5 and dicer1 are expressed in the epithelial tips while drosha and dgcr8 are basically absent. This dissimilar expression points toward the existence of a non-canonical miRNA pathway that most likely bypasses the microprocessor processing step of the canonical biosynthetic pathway. Actually, an alternative nuclear pathway for miRNA biogenesis, known as the mirtron pathway, was described in invertebrates (Okamura et al., 2007; Ruby et al., 2007). Subsequently, deep sequencing technology and computational methods have permitted the identification of mirtrons in mammals (Berezikov et al., 2007) and in chicken (Glazov et al., 2008). Mirtrons are pre-miRNA short introns that use splicing machinery to generate miRNA hairpins directly suitable for Dicer cleavage. In this sense, the requirement for Drosha/DGCR8 complex is circumvented and the mirtron pathway then merges with the canonical miRNA pathway at the Exportin-5-bound transport stage (Okamura et al., 2007). Taking into account the absence of drosha and dgcr8 in lung epithelium, and the existence of mirtrons in the chick embryo, it is plausible to think that in this compartment a non-canonical miRNA biosynthetic pathway might be active.

In conclusion, the mesenchymal and epithelial expression of microRNA processing machinery in developing chick lung support the previously recognized regulatory role of this mechanism in epithelial and mesenchymal morphogenesis. These findings also suggest a similar role for some specific miRNAs in the chick lung.

\section{ACKNOWLEDGMENTS}

The authors would like to thank Dr. Raquel P. Andrade for providing the probes used in this manuscript. We also acknowledge Luís Martins and Ana Lima for slide sectioning.

\section{REFERENCES}

Abdelfattah AM, Park C, Choi MY (2014) Update on non-canonical microRNAs. Biomol Concepts 5(4):275-87 
Banerjee A, Schambach F, DeJong CS, Hammond SM, Reiner SL (2010) Micro-RNA155 inhibits IFN-gamma signaling in CD4+ T cells. Eur J Immunol 40:225-231

Bartel DP (2004) MicroRNAs: genomics, biogenesis, mechanism, and function. Cell 116(2):281-297

Berezikov E, Chung WJ, Willis J, Cuppen E, Lai EC (2007) Mammalian mirtron genes. Mol Cell 28:328-336

Bernstein E, Kim SY, Carmell MA, Murchison EP, Alcorn H, Li MZ, Mills AA, Elledge SJ, Anderson KV, Hannon GJ (2003) Dicer is essential for mouse development. Nat Genet 35:215-217

Bhaskaran M, Wang Y, Zhang H, Weng T, Baviskar P, Guo Y, Gou D, Liu L (2009) MicroRNA-127 modulates fetal lung development. Physiol Genomics 37:268-278

Bhaskaran M, Xi D, Wang Y, Huang C, Narasaraju T, Shu W, Zhao C, Xiao X, More S, Breshears M, Liu L (2012) Identification of microRNAs changed in the neonatal lungs in response to hyperoxia exposure. Physiol Genomics 44:970-980

Carraco G, Gonçalves AN, Serra C, Andrade RP (2014) MicroRNA processing machinery in the developing chick embryo. Gene Expr Patterns 16(2):114-121

Carraro G, El-Hashash A, Guidolin D, Tiozzo C, Turcatel G, Young BM, De Langhe SP, Bellusci S, Shi W, Parnigotto PP, Warburton D (2009) miR-17 family of microRNAs controls FGF10-mediated embryonic lung epithelial branching morphogenesis through MAPK14 and STAT3 regulation of E-Cadherin distribution. Dev Biol 333(2):238-250

Carraro G, Shrestha A, Rostkovius J, Contreras A, Chao CM, El Agha E, Mackenzie B, Dilai S, Guidolin D, Taketo MM, Günther A, Kumar ME, Seeger W, De Langhe S, Barreto G, Bellusci S (2014) miR-142-3p balances proliferation and differentiation of mesenchymal cells during lung development. Development 141(6):1272-1281

Dakhlallah D, Batte K, Wang Y, Cantemir-Stone CZ, Yan P, Nuovo G, Mikhail A, Hitchcock CL, Wright VP, Nana-Sinkam SP, Piper MG, Marsh CB (2013) Epigenetic 
regulation of miR-17 92 contributes to the pathogenesis of pulmonary fibrosis. Am $\mathrm{J}$ Respir Crit Care Med 187(4):397-405

Dong J, Jiang G, Asmann YW, Tomaszek S, Jen J, Kislinger T, Wigle DA (2010) MicroRNA networks in mouse lung organogenesis. PLoS One 5(5):e1085

Glazov EA, Cottee PA, Barris WC, Moore RJ, Dalrymple BP, Tizard ML (2008) A microRNA catalog of the developing chicken embryo identified by a deep sequencing approach. Genome Res 18:957-964

Hamburger V, Hamilton HL (1992) A series of normal stages in the development of the chick embryo. Dev Dyn 195:231-272

Harris KS, Zhang Z, McManus MT, Harfe BD, Sun X (2006) Dicer function is essential for lung epithelium morphogenesis. Proc Natl Acad Sci 103(7):2208-2213

Henrique D, Adam J, Myat A, Chitnis A, Lewis J, Ish-Horowicz D (1995) Expression of a Delta homologue in prospective neurons in the chick. Nature 375:787-790

Herriges M, Morrisey EE (2014) Lung development: orchestrating the generation and regeneration of a complex organ. Development 141(3):502-513

Hogan BLM (1999) Morphogenesis. Cell 96:225-233

Jiang Z, Cushing L, Ai X, Lü J (2014) miR-326 is downstream of Sonic hedgehog signaling and regulates the expression of Gli2 and smoothened. Am J Respir Cell Mol Biol 51(2):273-283

Johanson TM, Lew AM, Chong MM (2013) MicroRNA-independent roles of the RNase III enzymes Drosha and Dicer. Open Biol 3(10):130144

Khoshgoo N, Kholdebarin R, Iwasiow BM, Keijzer R (2013) MicroRNAs and lung development. Pediatr Pulmonol 48(8):317-323 
Lü J, Qian J, Chen F, Tang X, Li C, Cardoso WV (2005) Differential expression of components of the microRNA machinery during mouse organogenesis. Biochem Biophys Res Commun 334(2):319-323

Lu Y, Thomson JM, Wong HY, Hammond SM, Hogan BL (2007) Transgenic overexpression of the microRNA miR-17-92 cluster promotes proliferation and inhibits differentiation of lung epithelial progenitor cells. Dev Biol 310(2):442-453

Metzger RJ, Klein OD, Martin GR, Krasnow MA (2008) The branching programme of mouse lung development. Nature 453:745-750

Moura RS, Carvalho-Correia E, daMota P, Correia-Pinto J (2014) Canonical Wnt signaling activity in early stages of chick lung development. PLoS ONE 9(3):e112388

Moura RS, Coutinho-Borges JP, Pacheco AP, daMota PO, Correia-Pinto J (2011) FGF signaling pathway in the developing chick lung: expression and inhibition studies. PLoS ONE 6(3):e17660

Mujahid S, Logvinenko T, Volpe MV, Nielsen HC (2013) miRNA regulated pathways in late stage murine lung development. BMC Dev Biol 13:13

Nana-Sinkam SP, Karsies T, Riscili B, Ezzie M, Piper M (2009) Lung microRNA: from development to disease. Expert Rev Respir Med 3(4):373-385

Okamura K, Hagen JW, Duan H, Tyler DM, Lai EC (2007) The mirtron pathway generates microRNA-class regulatory RNAs in Drosophila. Cell 130:89-100

Ouellet DL, Perron MP, Gobeil LA, Plante P, Provost P (2006) MicroRNAs in gene regulation: when the smallest governs it all. J Biomed Biotechnol 2006(4):69616

Ruby JG, Jan CH, Bartel DP (2007) Intronic microRNA precursors that bypass Drosha processing. Nature 448:83-87.

Sato T, Liu X, Nelson A, Nakanishi M, Kanaji N, Wang X, Kim M, Li Y, Sun J, Michalski J, Patil A, Basma H, Holz O, Magnussen H, Rennard SI (2010) Reduced miR146a 
increases prostaglandin $\mathrm{E}(2)$ in chronic obstructive pulmonary disease fibroblasts. Am J Respir Crit Care Med 182:1020-1029

Sayed D, Abdellatif M (2011) MicroRNAs in development and disease. Physiol Rev $91(3): 827-887$

Stefani G, Slack FJ (2008) Small non-coding RNAs in animal development. Nat Rev Mol Cell Biol 9(3):219-230

Warburton D, El-Hashash A, Carraro G, Tiozzo C, Sala F, Rogers O, De Langhe S, Kemp PJ, Riccardi D, Torday J, Bellusci S, Shi W, Lubkin SR, Jesudason E (2010) Lung organogenesis. Curr Top Dev Biol 90:73-158

Williams AE, Moschos SA, Perry MM, Barnes PJ, Lindsay MA (2007) Maternally imprinted microRNAs are differentially expressed during mouse and human lung development. Dev Dyn 236(2):572-580

Yanaihara N, Caplen N, Bowman E, Seike M, Kumamoto K, Yi M, Stephens RM, Okamoto A, Yokota J, Tanaka T, Calin GA, Liu CG, Croce CM, Harris CC (2006) Unique microRNA molecular profiles in lung cancer diagnosis and prognosis. Cancer Cell 9:189-198

Yang Y, Kai G, Pu XD, Qing K, Guo XR, Zhou XY (2012) Expression profile of microRNAs in fetal lung development of Sprague-Dawley rats. Int J Mol Med 29(3):393402

\section{FIGURE CAPTIONS}

Fig 1 drosha expression pattern in early stages of chick lung development. Representative examples of whole mount in situ hybridization (a-c) of stage b1, b2 and b3, sections of hybridized lungs (d-f) and correspondent HE stained sections ( $g-i)$. Dark arrowhead: dorsal mesenchyme. Open arrowhead: distal mesenchyme. Asterisk: distal epithelium. Black arrow: secondary bronchi epithelium. The black and dashed 
rectangles indicate the coronal and axial region, respectively, shown in the corresponding slide sections. Scale bars: $a / c-2000 \mu \mathrm{m} ; \mathrm{d} / \mathrm{i}-100 \mu \mathrm{m}$.

Fig 2 dgcr8 expression pattern in early stages of chick lung development. Representative examples of whole mount in situ hybridization (a-c) of stage b1, b2 and b3, sections of hybridized lungs (d-f) and correspondent HE stained sections $(g-i)$. Dark arrowhead: dorsal mesenchyme. Open arrowhead: distal mesenchyme. Asterisk: distal epithelium. Black arrow: secondary bronchi epithelium. The black and dashed rectangles indicate the coronal and axial region, respectively, shown in the corresponding slide sections. Scale bars: $a / c-2000 \mu \mathrm{m} ; \mathrm{d} / \mathrm{i}-100 \mu \mathrm{m}$.

Fig 3 exportin-5 expression pattern in early stages of chick lung development. Representative examples of whole mount in situ hybridization (a-c) of stage b1, b2 and b3, sections of hybridized lungs (d-f) and correspondent HE stained sections ( $g-i)$. Dark arrowhead: dorsal mesenchyme. Open arrowhead: distal mesenchyme. Asterisk: distal epithelium. Black arrow: secondary bronchi epithelium. The black and dashed rectangles indicate the coronal and axial region, respectively, shown in the corresponding slide sections. Scale bars: $a / c-2000 \mu \mathrm{m} ; \mathrm{d} / \mathrm{i}-100 \mu \mathrm{m}$.

Fig 4 dicer1 expression pattern in early stages of chick lung development. Representative examples of whole mount in situ hybridization (a-c) of stage b1, b2 and b3, sections of hybridized lungs (d-f) and correspondent HE stained sections ( $g-i)$. Dark arrowhead: dorsal mesenchyme. Open arrowhead: distal mesenchyme. Asterisk: distal epithelium. Black arrow: secondary bronchi epithelium. The black and dashed rectangles indicate the coronal and axial region, respectively, shown in the corresponding slide sections. Scale bars: $a / c-2000 \mu \mathrm{m} ; \mathrm{d} / \mathrm{i}-100 \mu \mathrm{m}$. 